Instability, Chaos and Predictability in Celestial Mechanics and Stellar Dynamics
Copyright $\odot 1992$ by Nova Science Publishers, Inc. All rights of reproduction in any form reserved.

ISBN 1-56072-054-9

\title{
NEW INTERMEDIARIES FOR THE MAIN PROBLEM IN SATELLITE THEORY
}

\author{
José M. Ferrándiz and Luis Floría \\ Departamento de Matemática Aplicada a la Ingeniería, E.T.S. de Ingenieros \\ Industriales, Universidad de Valladolid, 47011 Valladolid, Spain
}

ABSTRACT. After reviewing the original approach leading to the introduction of intermediaries in Satellite Theory, a general procedure to define intermediaries for the Main Problem in this Theory is proposed. This procedure is susceptible to an intuitive interpretation analogous to solving a simple puzzle. The application of this method to the Main Problem allows us not only to recover the well known classical intermediaries but al so to obtain several completely new ones, all admitting simple solutions.

\section{REVIEWING CLASSICAL INTERMEDIARIES}

Criteria and ideas leading to the definition of intermediaries for the Main Problem in Satellite Theory have undergone substantial modifications since their introduction by Sterne ([29],[30]) and Garfinke1 ([17],[18],[19]) until the papers by Deprit [13] and Deprit and Ferrer [14]. The nature of these modifications is twofold, and is related to the consideration of the two following aspects: the basic mathematical technique put into action (first, the Hamilton-Jacobi Equation; later, infinitesimal canonical transformations expanded in powers of a small parameter), and the way in which certain disposable parameters are treated (as absolute constants or, afterwards, as constants of the motion). The fundamental milestones in this evolution and the previously existing theoretical background will next be considered in a brief review.

Outstanding precedents in the use of more general orbits than a conventional Keplerian ellipse as the starting point of a process of successive approximations can be traced back to Hill's investigations ([25],[26]) concerning the Moon's motion and Brown's studies $([6],[7],[8])$ in Lunar Theory. 
Gylden ([22]) had also applied this technique as an approximate method in the three-body problem, and particularly in studying Lunar motion ([ 23]).

Prior to the launch of artificial satellites Brouwer ([2],[3]) had al ready undertaken the research of the motion of a particle of negligible mass subjected to the gravitational attraction of a spheroid.

Brouwer himself $([4],[5, \mathrm{Ch} .17, \mathrm{Sec}, 12],[24, \mathrm{Ch} .9$, sec. 9.20]) also considered the Main Problem in Satellite Theory and built up an important analytical theory that henceforth became a necessary point of reference for a great number of researchers. This theory yields a Keplerian uniformly precessing ellipse later taken as the unperturbed orbit in the Delaunay Theory, i.e., as the basis of subsequent developments. Such an ellipse could be regarded as related to a hidden, not explicitly mentioned, intermediary.

In a parallel way, theories based on the use of intermediary Hamiltonians for the Main Problem ([13],[21]) were developed. The definitions of the first classical intermediaries in Satellite Theory arose in a very short interval of time; they were conceived as simplified problems supplying mere approximations to the real motion of a satellite and were inspired by the requirement that the Hamilton-Jacobi Equation attached to the intermediary Hamiltonian would turn out to be separable (thus providing a set of angle-action variables for the problem described by the intermediary) or at least integrable, and that the intermediary would contain as many as possible of the secular effects of the first-order perturbations due to terms present in the Main Problem.

In this way Sterne $([29],[30])$ and Garfinkel ([17],[18], [19]) constructed their approximations to the secular motion of satellites with the help of potentials that in spherical polar co-ordinates could be solved by separation of variables ([ 5, p.592], [ $28, \mathrm{Ch} .10, \mathrm{Sec}, 10.5]$ ) and involved parameters treated as absolute constants, determined in such a way as to minimize the number of elliptic functions appearing in the solution and to remove the first-order secular variations-when residual perturbations were added to the intermediary-in the intermediate orbit. However, resorting to absolute constants raises great difficulties when trying to extend the theory to higher orders.

Garfinkel also specified ([19],[20],[21]) requirements for the most general potential that he would accept in an intermediary, and improved his previous theory $([20],[24$, Ch. 9, sec. 9.21]) by adjusting the disposable constants of his 1958 intermediate orbit so as to incorporate into the intermediary the secular variations up to the second order obtained in his 1959 study.

An essential change in the nature of the approach to the construction of intermediaries is due to Aksnes, who defined 
a new intermediary by treating formally as a dynamical variable the available parameter appearing in his potential and requiring that the only first-order perturbations suffered by the solution of the Hamiltonian be purely periodic. His choice of the parameter as a variable under perturbations allowed him to determine it so as to make the secular part of the disturbing potential be identically zero, and enabled him ([1]) to build up a complete analytical second-order theory in Hill variables using Hori's perturbation method ([27]). The intermediaries developed by Sterne, Garfinkel and Aksnes: were compared in [21] and considered as members of a four-parameter family of intermediaries.

However, as far as we know, nothing had ever been said about the possibility of defining intermediaries as a result of applying a canonical transformation, admitting (at least at the first order) a periodic generating function, to the Main Problem. Ideas of this nature do not appear in the 1 iterature until the publications by $C i d$ and Lahul1a ([9],[11]), who obtained the first radial intermediary as a result of an infinitesimal contact transformation applied to the Main Problem to eliminate the latitude through the Pincare-von Zeipel method, and later $([10])$ by means of the Lie transform technique as presented by Deprit in [12]. This approach was qualitatively new in Satellite Theory, and constitutes a breakthrough in the way in which the intermediaries were conceived. Subsequently, in the study of satellite motion the Hamilton-Jacobi Theory gave ground to canonical perturbation methods based on the use of Lie series.

In his 1981 paper [13], and again in the context of Lie transformations, Deprit systematized the previously existing intermediaries, derived the simplest radial intermediary (in fact, he constructed a new family of radial intermediaries), and introduced new concepts establishing the distinction between common versus natural intermediaries, and zonal versus radial intermediaries, the latter being very simple to handle. In his revision of previous classical intermediaries Deprit did not use the original variables in which their authors had formulated them; the expressions given by Deprit involve the resulting variables after applying a transformation like the Lie canonical one, called by him the elimination: of the parallax, to the Main Problem. Moreover, he extended Sterne's and Garfinkel's definitions, using constants of the motion instead of absolute constants.

For a display of the expressions of the above-mentioned classical intermediaries, the reader is refered to the Appendix in the final section of the present paper.

\section{OUTLINES OF A METHOD TO DEFINE INTERMEDIARIES}

In this section we propose a general procedure to generate intermediaries. This procedure allows us to obtain all 
the intermediaries already known in spherical coordinates and some more new ones, all with simple solutions. A thorough analysis of the existing intermediaries shows that, at the first order, they can be generated in a simple and systematic manner, similar to that of solving a puzzle. The basic idea consists of replacing a term in the Hamiltonian of the Main Problem by another expression giving the same average along solutions to the unperturbed problem and such that the resulting Hamiltonian is separable in a certain chart.

To start with, we split up the disturbing part of the Main Problem into sufficiently manageable "pieces". We next replace one or several of them by another one "fitting properly" into the place of the former: it will happen when both parts, old and new, have the same average along the unperturbed motion. Such a replacement admits an easy mathematical interpretation: we can consider that the new "piece" is added and subtracted in the expression of the Hamiltonian corresponding to the Main Problem, and the difference between the old and the new part - which is periodic in the angles involved in the solution to the unperturbed problem - is then eliminated by means of a Lie transformation or any other perturbation method. We must finally check that the result is indeed an intermediary. To this end we shall require that the new Hamiltonian be integrable or separable in some chart, this last requirement being more easily manageable in practice.

This idea of the puzzle has already been suggested for the first time in a previous paper [15],[16] by the authors, although fewer "pieces" were used on that occasion. In the present paper we remove this limitation and apply the procedure to obtain a set of intermediaries (that could be considered as the fundamental ones) in Hill variables; among them some completely new ones, not known hitherto, will appear. of course the use of other sets of variables could lead to other different intermediaries.

In what follows we aim to establish the notations and fundamentals pieces that we shall bring into play in order to construct intermediaries, i.e., in order to solve the aforementioned puzzle. With the purpose of making the comparisons easy, we shall adopt notations close to those used by Deprit [13]. In the well-known canonical set $(R, \theta, N ; r, \theta, \nu)$ of Hill's polar-nodal variables with $\theta$ denoting the argument of latitude, $v$ being the argument of the ascending node and $\cos I=N / \odot$, the Main Problem in Satellite Theory is described by the Hamiltonian function.

where

$$
\begin{array}{r}
H=H_{0}+\varepsilon H_{1} \\
H_{0}=\frac{1}{2}\left(R^{2}+\frac{\Theta^{2}}{r^{2}}\right)-\frac{\mu}{r}, \epsilon H_{1}=\frac{\mu}{r}\left[J_{2}\left(\frac{R_{E}}{r}\right)^{2}\right. \\
\left.P_{2}(\sin I \sin \theta)\right],
\end{array}
$$


$R_{E}$ is the mean equatorial radius of the Earth, $\varepsilon=-\mu R_{E}^{2} J$, and $H_{0}$ represents an unperturbed Kepler problem.

Just 1 ike in our previous work $[15],[16]$ we shall split $\mathrm{H}_{1}$ into the form

with

$$
\mathrm{H}_{1}=\mathrm{AP}_{3}+\mathrm{BQ}_{3} \text {, }
$$

$$
\begin{array}{ll}
A=\frac{3 \cos ^{2} I-1}{4}, & B=\frac{3 \sin ^{2} I}{4}, \\
\mathrm{P}_{3}=\frac{1}{\mathrm{r}}, & \mathrm{Q}_{3}=\frac{1}{\mathrm{r}^{3}} \cos 2 \theta .
\end{array}
$$

Making use of the standard notations for the Keplerian elements, after straightforward calculations we ascertain that the functions

$$
\tilde{\mathrm{p}}_{2}=\frac{1}{\mathrm{pr}^{2}}, \tilde{\mathrm{p}}_{1}=\frac{1}{\mathrm{p}^{2} \mathrm{r}} \sqrt{\frac{\mathrm{\mu}}{\mathrm{a}}} \text { and } \tilde{\mathrm{p}}_{\mathrm{o}}=\frac{\mathrm{n}}{\sqrt{\mu} \mathrm{p}^{3 / 2}} \text {, }
$$

where $p$ is the semi-1atus rectum and $n=\mu^{1 / 2} a^{-3 / 2}$ denotes the mean motion, have the same average as $P_{3}$, these averages being calculated as integrals over the time around a closed path.

Given that $Q_{3}$ averages out to zero, very numerous options can be taken as the candidates for replacing $Q_{3}$. However, in view of the fact that we have restricted ourselves to requiring separability in Hill variables of the intermediary and bearing in mind the proposition quoted by Deprit in $[13, p .119]$, the number of substitutes for $Q_{3}$ reduces to the following two:

$$
\tilde{Q}_{2}-\frac{1}{\mathrm{pr}^{2}} \cos 2 \theta, \quad \tilde{Q}_{0}=0 \text {. }
$$

Observe that the preceding functions could be used to define intermediaries in a very rudimentary way, by considering $\mathrm{p}, \mathrm{a}, \mathrm{n}$ to be absolute constants, though the seeming simplicity of this procedure would lose its advantages when attempting to carry the theory to higher orders, as happened in Garfinkel's theory.

Our approach requires that all three parameters $p, a, n$ should be expressed in terms of canonical variables (as first done by Aksnes). The choice of $p=\theta^{2} / \mu$ is obvious. As for $a$ and $n$ we can make use of the relations

$$
a=\frac{\mu}{-2 h} \text { and } n=\frac{(-2 h)^{3 / 2}}{\mu}
$$


Now we can decide on replacing $h$ by the expression of the Keplerian energy in Hill variables, i.e., $h=H_{0}(R, \theta, r)$, which would lead to involved developments. We prefer to work in extended phase space $\left(R, \theta, N, P_{0} ; r, \theta, v, t\right)$, in which $P_{0}=-h$ is an additional canonical variable.

Taking into account the preceding ideas, the pieces to be used in the puzzle are

$$
\begin{aligned}
& P_{2}=\frac{1}{\mathrm{pr}^{2}}, \mathrm{P}_{1}=\frac{\mu}{\mathrm{r} \theta^{3}} \sqrt{2 \mathrm{P}_{0}}, \mathrm{P}_{0}=\frac{\left(2 \mathrm{P}_{0}\right)^{3 / 2}}{\theta^{3}} \\
& \mathrm{Q}_{2}=\frac{1}{\mathrm{pr}^{2}} \cos 2 \theta, \mathrm{Q}_{0}=0,
\end{aligned}
$$

where $p=\theta^{2} / \mu$.

Let us notice that this way of acting is in line with that widely followed in studies related to DS (DelaunaySimilar) variables.

\section{LIST OF INTERMEDIARIES}

Completing the 1 ist of intermediaries which are separable in Hill variables is now reduced to a combinatorial problem. In all we have $4 \times 2=8$ possibilities at our disposal; they are displayed and designated by $\mathrm{H}_{(\mathrm{ij})}$, where the subscripts refer to the ordered pair $\left(P_{i}, Q_{j}\right)$ of pieces involved in the corresponding construction.

The known intermediaries are:

$\left(P_{3}, Q_{2}\right)$ : Sterne's Zonal Intermediary:

$$
\mathrm{H}_{(3,2)}=\frac{\mathrm{A}}{\mathrm{r}^{3}}+\frac{\mathrm{B}}{\mathrm{pr}} \cos 2 \theta \text {; }
$$

$\left(P_{2}, Q_{2}\right)$ : Aksnes' Zonal Intermediary:

$$
\mathrm{H}_{(2,2)}=\frac{\mathrm{A}}{\mathrm{pr}^{2}}+\frac{\mathrm{B}}{\mathrm{pr}} \cos 2 \theta \text {; }
$$

$\left(P_{3}, Q_{0}\right):$ Cid-Lahulla's Radial Intermediary:

$$
\left.H_{(3,0}\right)=\frac{A}{r^{3}} \text {; }
$$

$\left(\mathrm{P}_{2}, \mathrm{Q}_{0}\right):$ Deprit's Radial Intermediary:

$$
\mathrm{H}_{(2,0)}=\frac{\mathrm{A}}{\mathrm{pr}^{2}}
$$


Let us stress that Sterne did not actually use the Intermediary that we have displayed, since his constants were taken to be absolute constants; however we shall term it after him, as done by Deprit.

The new intermediaries, some radial and others zonal, are

$$
\begin{aligned}
& \left(P_{1}, Q_{0}\right): H(1,0)=\frac{A \sqrt{2 P_{0}}}{r^{3} / \mu} ; \\
& \left(P_{1}, Q_{2}\right): H(1,2)=\frac{A \sqrt{2 P_{0}}}{r \theta^{3} / \mu}+\frac{B}{\mathrm{pr}^{2}} \cos 2 \theta ; \\
& \left(P_{0}, Q_{0}\right): H(0,0)=\frac{A\left(2 P_{0}\right)^{3 / 2}}{\theta^{3}} ; \\
& \left(P_{0}, Q_{2}\right):\left(H_{(0,2)}=\frac{A\left(2 P_{0}\right)^{3 / 2}}{\theta^{3}}+\frac{B}{\mathrm{pr}^{2}} \cos 2 \theta\right.
\end{aligned}
$$

The first one in this list was also the first intermediary found by the first author, and can be considered as the origin for a new family of radial intermediaries that share, with those introduced by Deprit, a great proximity to Keplerian motion. In fact it allows a simple solution in closed form that will be published in a further paper dealing with radial intermediaries in a wider framework.

The intermediary defined by $H_{(0,0)}$ from the pair $\left(P_{0}, Q_{0}\right)$ is close to the secular Hamiltonian of Brouwer's solution, al though not exactly the same due to the dependence prescribed for the semi-major axis.

Let us mention the fact that other valid intermediaries can be obtained by combining those previously 1 isted in expressions of the form

$$
\sum \lambda_{i} P_{i}+\sum \mu_{j} Q_{j},
$$

and $\lambda_{i}$ and $\mu_{j}$ being constants subject to the restriction $\Sigma \lambda_{i}=1$. In so doing, Hamiltonians of the form

$$
H_{\text {MIXED }}=H_{0}+E\left\{A \Sigma \lambda_{i} P_{i}+B \Sigma \mu_{j} Q_{j}\right\}
$$

could be obtained.

A Garfinkel-1ike intermediary could be included in this last group of mixed intermediaries, although its derivation 
is rather complex because its author introduced terms depending on an inclination taken as an absolute constant. This difficulty could be circumvented by modifying its definition, e.g., by using

$$
H_{1}^{\prime}=A\left(3 P_{2}-2 P_{1}\right)+B Q_{2}
$$

In this way, the generating function giving the modified Garfinkel Intermediary

$$
\mathrm{H}_{\mathrm{G}}^{\prime}=\mathrm{H}_{0}+\varepsilon \mathrm{H}_{1}^{\prime}
$$

is periodic. In relation to this subject we would point out that the version presented by Deprit is different from the extension obtained by our procedure.

Let us finally point out that the "pieces" involved in our construction of intermediaries only contain powers of $r$ with the exponents $0,1,2$ and 3 . After the above considerations, and by virtue of proposition [13,p.119], any arbitrary power of $r$ could be used when applying the procedure that we have described.

\section{CONCLUDING REMARKS}

We have presented a general procedure to obtain intermediaries; it has been applied to increase the family of those which are separable in Hill variables. As a result of that, some new intermediaries have come to 1 ight. We would like to pay special attention to a radial one of very simple expression.

Additional aspects related to the study of these and other questions will be treated in forthcoming papers. Let us say at this moment that, as expected, the behaviour of the new intermediaries that we have presented here is very similar.

The ideas developed in this paper are also applicable to the analysis of more general problems than the Yain Problem. So the method could be used to define more intermediaries (even for the Main Problem) or for the treatment of other problems in Celestial Mechanics.

\section{APPEND IX}

We next set down the expressions of the Main Problem and the known intermediaries. The subscripts refer to the name of the author who introduced the corresponding intermediary. The notations are close to those used in the original papers; the planetocentric declination of the satellite with respect to the planet's equator is represented by $\beta$, and satisfies the relation $\sin \beta=\sin I \sin \theta, I$ being the inclination of the orbit, and $\theta$ denotes the argument of latitude; here $B$ is not the same as in Section 2 . 
Main Problem:

$$
\begin{aligned}
H_{M} & =H_{0}+\varepsilon\left[\frac{1}{2}\left(3 \sin ^{2} \beta-1\right)\right] \frac{1}{r^{3}} \\
& =H_{0}+\varepsilon\left\{\frac{1}{r^{3}} \frac{\left(3 \cos ^{2} I-1\right)}{4}+\frac{1}{r^{3}} \frac{3 \sin ^{2} I}{4} \cos 2 \theta\right\},
\end{aligned}
$$

where $\mathrm{H}_{0}$ is the Hamiltonian corresponding to an unperturbed Kepler problem.

\section{Sterne's Intermediary:}

Add the term

$$
V_{1}=\varepsilon\left\{\frac{3}{2} \sin ^{2} B-\frac{3}{4} \sin ^{2} I_{S}\right\}\left\{\frac{1}{r^{3}}-\frac{1}{a_{S} r^{2}}\right\}
$$

to the Main Problem, where $I_{S}$ (the maximum declination) and $a_{s}$ (the mean distance are absolute constants. Then $H_{S}^{\prime}=H_{0}-\varepsilon \frac{1}{a_{S} r^{2}}\left\{\frac{3}{2} \sin ^{2} B-\frac{3}{4} \sin ^{2} I_{S}\right\}-\varepsilon \frac{1}{r^{3}}\left\{-\frac{1}{2}+\frac{3}{4} \sin ^{2} I_{S}\right\}$

\section{Sterne's Intermediary:}

$$
\begin{array}{r}
H_{S}=H_{0}-\varepsilon \frac{1}{p_{S} r^{2}}\left\{\frac{3}{2} \sin ^{2} B-\frac{3}{4} \sin ^{2} I_{S}\right\} \\
-\varepsilon \frac{1}{r^{3}}\left\{-\frac{1}{2}+\frac{3}{4} \sin ^{2} I_{S}\right\},
\end{array}
$$

with $p_{S}=a_{S}\left(1-e_{S}^{2}\right)$.

1958 Garfinke1's Intermediary:

$$
\begin{aligned}
\mathrm{H}_{\mathrm{G}}=\mathrm{H}_{0}+ & \varepsilon \frac{3}{\mathrm{r}}\left\{1-\frac{3}{2} \sin ^{2} \mathrm{I}_{\mathrm{G}}\right\} \frac{1}{\mathrm{a}_{\mathrm{G}}^{2}\left(1-e_{\mathrm{G}}^{2}\right)^{3 / 2}} \\
& +\varepsilon\left(3 \sin ^{2} \beta-3 \cos ^{2} \mathrm{I}_{\mathrm{G}}\right) \frac{1}{\mathrm{p}_{\mathrm{G}} \mathrm{r}^{2}}
\end{aligned}
$$

with $I_{G}, a_{G}, e_{G}$ and $p_{G}=a_{G}\left(1-e_{G}^{2}\right)$ absolute constants.

1959 Garfinke1's general results for intermediaries ( 19 , p.353], $[20$, p.224],[21,p.85]):

$H=H_{0}+\varepsilon 3\left\{c_{1}\left(\sin ^{2} B-c_{2}\right) \frac{1}{2 r^{2}}+\frac{c_{3}}{r}+\frac{c_{4}}{r^{3}}\right\}$ 
is the most general Hamiltonian such that it

- preserves the gross features of the Main Problem;

- separates the Hamilton-Jacobi Equation (in spherical coordinates);

- leads to a closed solution in terms of elliptic integrals;

- allows a proper choice of constants $c_{i}$ that incorporates into $H$ the whole first order secular variations of the Main Problem.

Aksnes' Intermediary:

$$
\mathrm{H}_{\mathrm{A}}=\mathrm{H}_{0}+\varepsilon\left[\frac{3}{2} \sin ^{2} \mathrm{I} \sin ^{2} \theta-\frac{1}{2}\right\} \frac{1}{\mathrm{pr}^{2}}
$$

where $c_{1}=1 / p$, with $p=\theta^{2} / \mu$, is to be treated as a dynamical variable when perturbations are applied.

The disposable parameter $c_{1}$ is determined to reduce the secular part of

$$
H_{M}-H_{A}=\varepsilon P_{2}(\sin B)\left\{-\frac{1}{3}-\frac{c_{1}}{r^{2}}\right\}
$$

identically to zero. Remember that $P_{2}(\sin \beta)$ is the Legendre polynomial of degree 2 in $\sin B$.

Cid-Lahul1a's Intermediary:

New ideas lead to their Intermediary

$$
\mathrm{H}_{\mathrm{C}}=\mathrm{H}_{0}+\varepsilon\left\{\frac{3 \cos ^{2} \mathrm{I}-1}{4}\right\} \frac{1}{\mathrm{r}^{3}} \text {, }
$$

obtained as a result of the elimination of the argument of latitude in the Main Problem by means of the von Zeipel Method.

\section{Deprit's Intermediary:}

In his 1981 paper [ 13] he obtained his Hamiltonian a (a family of them) by means of a lie transformation. These Hamiltonians can be defined up to an arbitrary order. At the first order one has

$\mathrm{H}_{\mathrm{D}}=\mathrm{H}_{\mathrm{O}}+\varepsilon\left\{\frac{3 \cos ^{2} \mathrm{I}-1}{4}\right\} \frac{1}{\mathrm{pr}^{2}}=\mathrm{H}_{0}+\varepsilon\left\{\frac{1}{2}-\frac{3}{4} \sin ^{2} \mathrm{I}\right\} \frac{1}{\mathrm{pr}^{2}}$,

with $\mathrm{p}=\theta^{2} / \mu$.

In notations close to those used by Deprit, the preceding intermediaries take the form

$J_{S}=H_{0}+\varepsilon\left\{\left[\frac{1}{2}-\frac{3}{4} \sin ^{2} I\right] \frac{1}{p^{2}}+\frac{3}{4} \sin ^{2} I \cos 2 \theta \frac{1}{r^{3}}\right\} ;$ 


$$
\begin{gathered}
\mathrm{J}_{\mathrm{G}}=\mathrm{H}_{0}+\varepsilon\left\{3\left[\frac{1}{2}-\frac{3}{4} \sin ^{2} \mathrm{I}\right] \frac{\mathrm{n}}{\mathrm{pr}^{2}}+\frac{3}{4} \sin ^{2} \mathrm{I} \cos 2 \theta \frac{1}{\mathrm{r}^{3}}\right\} ; \\
\mathrm{J}_{\mathrm{A}}=\mathrm{H}_{\mathrm{A}}=\mathrm{H}_{0}+\varepsilon\left\{\left[\frac{1}{2}-\frac{3}{4} \sin ^{2} \mathrm{I}\right] \frac{1}{\mathrm{pr}^{2}}+\frac{3}{4} \sin ^{2} \mathrm{I} \cos 2 \theta \frac{1}{\mathrm{pr}^{2}}\right\} ; \\
\mathrm{J}_{\mathrm{C}}=\mathrm{H}_{\mathrm{C}}=\mathrm{H}_{0}+\varepsilon\left\{\frac{1}{2}-\frac{3}{4} \sin ^{2} \mathrm{I}\right\} \frac{1}{\mathrm{r}^{3}} ; \\
\mathrm{J}_{\mathrm{D}}=\mathrm{H}_{\mathrm{D}}=\mathrm{H}_{0}+\varepsilon\left\{\frac{1}{2}-\frac{3}{4} \sin ^{2} \mathrm{I}\right\} \frac{1}{\mathrm{pr}^{2}} .
\end{gathered}
$$

Notice that $\mathrm{J}_{S}$ and $\mathrm{J}_{G}$ are not the same as $\mathrm{H}_{S}$ and $\mathrm{H}_{G}$. These last expressions for the intermediaries can be derived from the Main Problem by means of Lie transformations, the generating functions (except that leading to $J_{G}$ ) being periodic (up to the first order).

\section{ACKNOWLEDGEMENT}

The authors acknowledge the financial support received from CICYT of Spain under Project ESP 88-0541.

\section{REFERENCES}

[1] Aksnes, K.: 1970, Astron. J. 75,1066-1076.

[2] Brouwer,D.: 1946, Astron. J. 51, 223-231.

[3] Brouwer, D.: 1958, Astron. J.63,433-328.

[4] Erouwer, D.: 1959, Astron. J.64,378-397.

[5] Brouwer,D. and Clemence, G.M.: 1961, Methods of Celestial Mechanics, Acadenic Press, New York and London.

[6] Brown, E.W.: 1892, Amer.J. Math. 14,141-160.

[7] Brown, E. .:1893, Amer.J. Math. 15, 244-263.

[8] Brown, E.W.: 1895, Amer. J.Math.17, 318-358.

[9] Cid, R. and Labulla, J.F.: 1969, "Perturbaciones ed corto periodo en el movimiento de un satelite artificial, en function de las variables de Hill", Rev. Acad.Ciencias Zaragoza Serie $2^{\alpha}, 24,159-165$.

[10]Cid,R. and Lamul1a, J.F.: 1971, "Apl icacion declas transformaciones de Lie a la eliminacion de terminos de corto perlodo", Vrania, 274,177-184.

[11]Cid, R. and Lahulla, J.F.: 1971, "Perturbaciones de segundo orden y corto periodo, para el movimiento de un satelite artificial, en las variables de Hill", Rev.Acad. Ciencias Zaragoza, Serie $2^{\alpha}, 26,333-343$.

[12]Deprit, A.: 1969, Celest. Mech. 1,12-30. 
[13] Deprit, A.: 1981, Cel est.Mech. 24,111-153.

[14] Deprit, A. and Ferrer, S.: 1987, Celest. Mech.40,335343.

[15] Ferrandiz, J.M. and Floria, L.: 1989, "Generacion sistematica de intermediarios en la Teoria del Satelite", Actas de las XIV Jornadas Hispano-Lusas de Matematicas, Vol. III, $1207-1211$.

[16] Ferrandiz, J.M. and Floria, L.: 1989-1990, Towards a Systematic Definition of Intermediaries in the Theory of Artificial Satellites (Unpubl ished manuscript).

[17] Garfinkel, B.:1958, A stron. J.63,88-96.

[18] Garfinkel, B.:1959, A stron. J.64, 270-272.

[19] Garfinkel, B.:1959, A stron. J.64,353-367.

[20] Garfinkel, E.:1964, Astron. J.69,223-229.

[21] Garfinkel, B. and Aksnes, K.: 1970 , Astron. J.75,85-91.

[22] Gylden, H.:1882-1883, Acta Mathematica 1, 77-92.

[23] Gylden, $H_{0}:$ 1885-1886, Acta Mathematica 7, 125-172.

[24] Hagihara, Y.: 1971, Celestial Mechanics, Vol. II, Part I (Perturbation Theory), MIT Press.

[25] Hill, G.W.: 1878, Amer.J. Math. 1,pp. 5-26, 129-147, 245-: 260.

[26] Hili, G.W.: 1886, Acta Mathematica 8,1-36.

[27] Hri, G.-i.: 1966, Publ . A stron. Soc. Japan 18, 287-296.

[28] Roy, A.E.: 1988, Orbital Motion (Third Edition), Adam Hilger, Bristol and Philadelphia.

[29] Sterne, T.E.: 1957, Astron. J.62, 96 .

[30] Sterne, T.E.:1958, Astron. J. 63,28-40. 